\title{
Pt-on-Pd Dendritic Nanosheets with Enhanced Bifunctional Fuel Cell Catalytic Performance
}

Xiuying Peng ${ }^{\dagger,{ }^{\dagger}}$, , Dongtao Lu ${ }^{\dagger, \S}$, Yingnan Qin ${ }^{\ddagger}$, Miaomiao Li ${ }^{\dagger}$, Yujing Guo ${ }^{\dagger} *$,

Shaojun Guo ${ }^{+* *}$

$\dagger$ Institute of Environmental Science, Shanxi University, Taiyuan, Shanxi, 030006, PR China

\$ Department of Materials Science and Engineering, College of Engineering, Peking University, Beijing, 100871, PR China

$\S$ Equal contribution

* Corresponding author: guoyj@ sxu.edu.cn, guosj@pku.edu.cn 

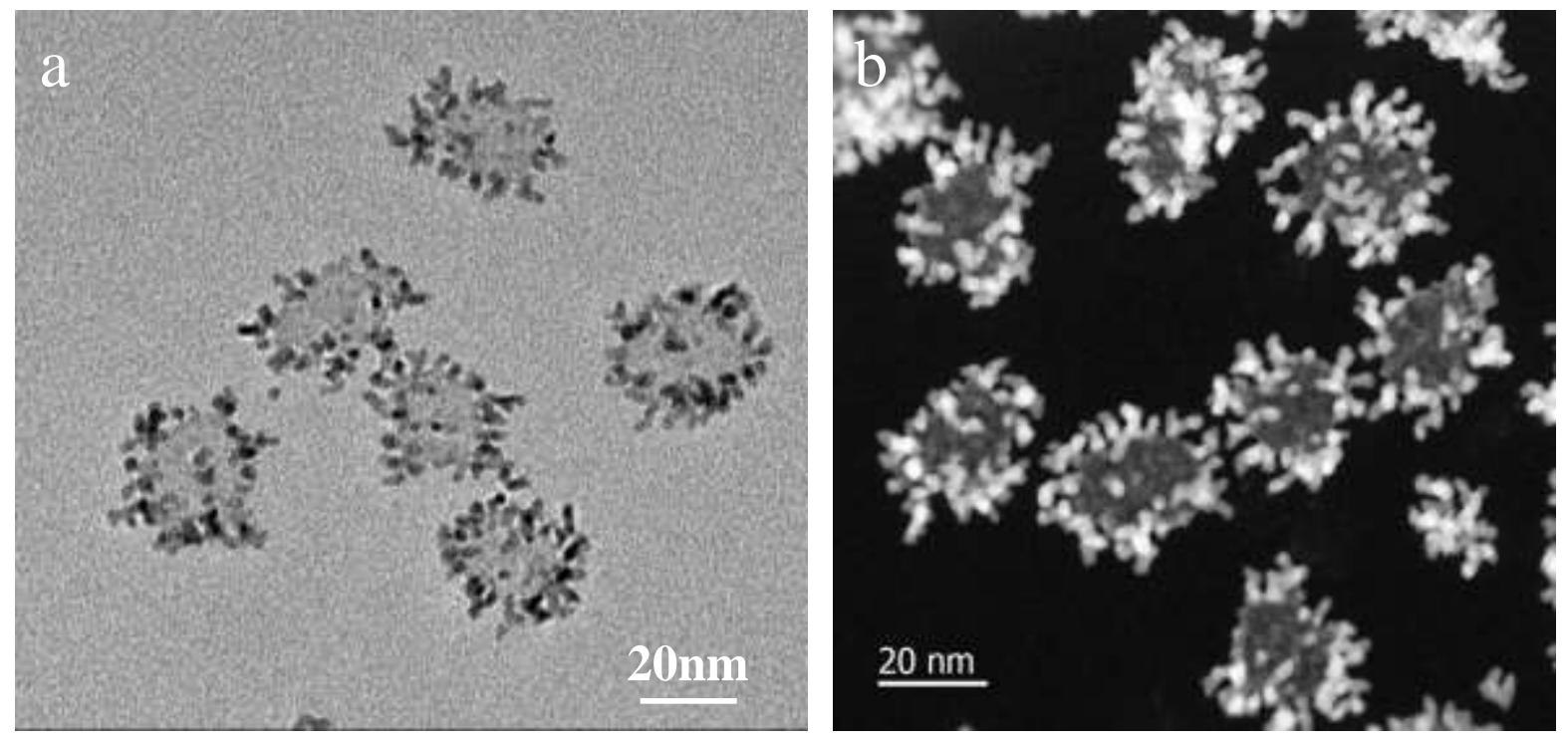

Figure S1 (a) Low-magnification TEM image and (b) Low-magnification HAADF-STEM image of $\mathrm{Pd}_{1} \mathrm{Pt}_{4}$ DNSs. 

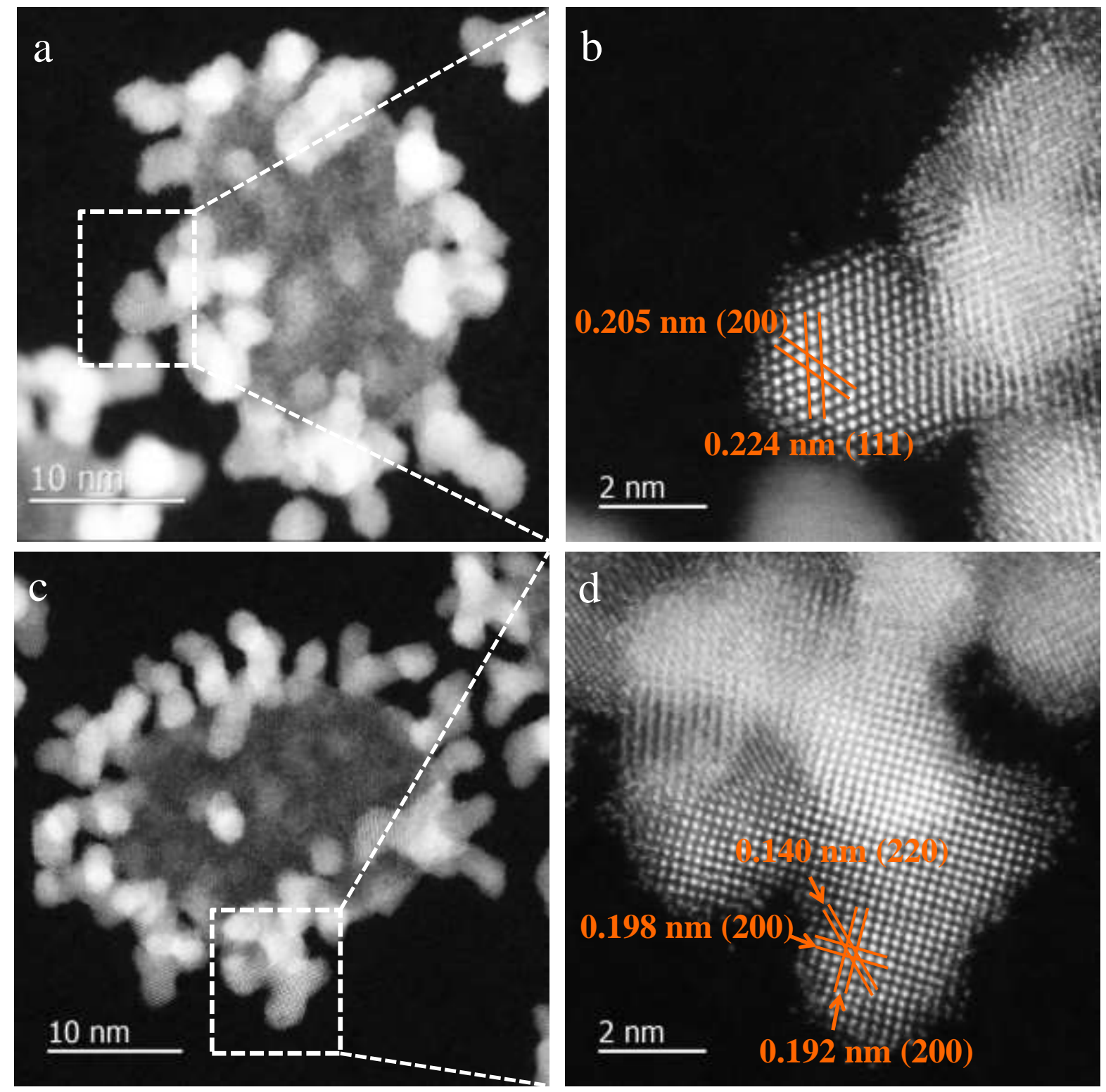

Figure S2 (a, c) Low- and (b, d) High-magnification HAADF-STEM images of $\mathrm{Pd}_{1} \mathrm{Pt}_{4}$ DNSs. 

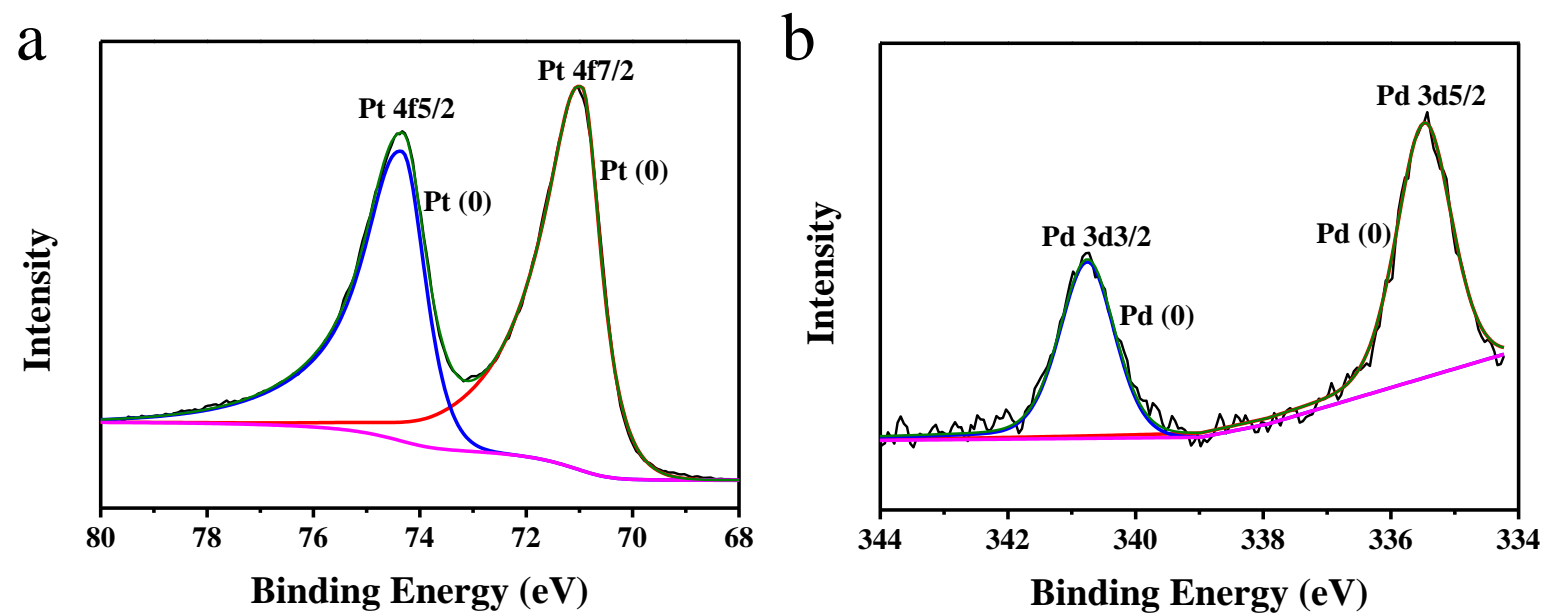

Figure S3. XPS spectroscopy of $\mathrm{Pd}_{1} \mathrm{Pt}_{4}$ DNSs (a) Pt4f; (b) Pd3d. 

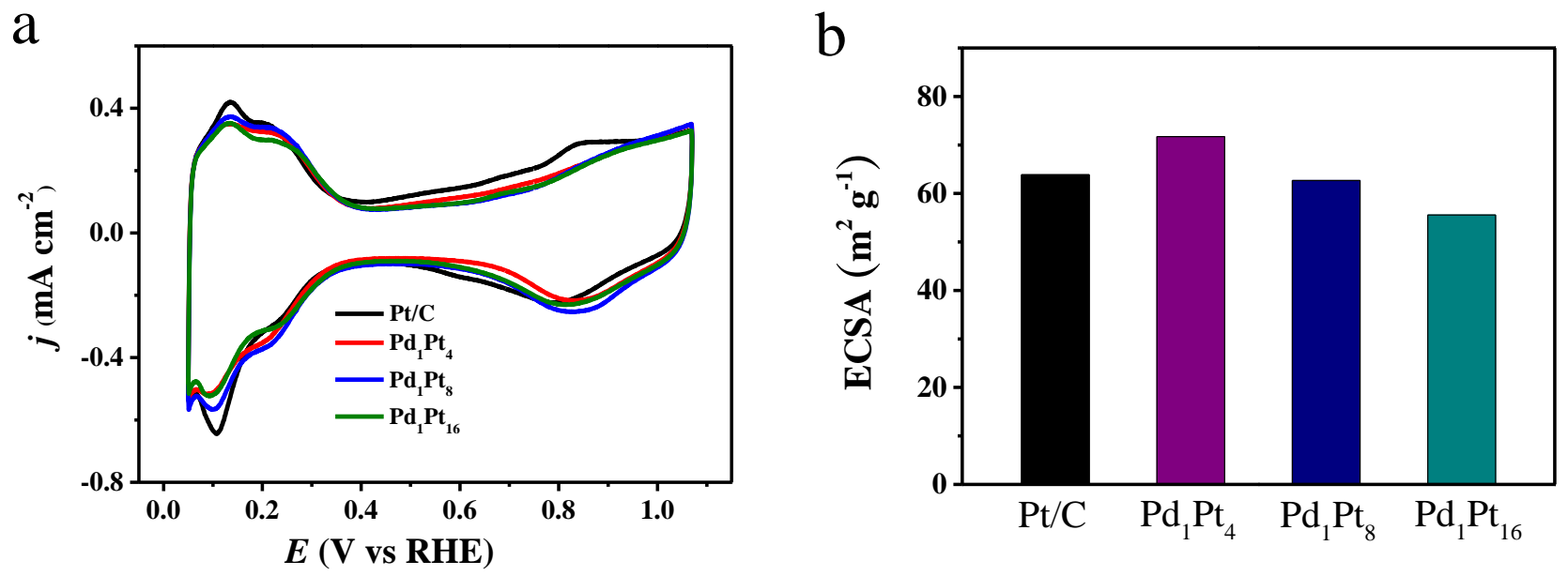

Figure S4. (a) CVs of various catalysts in a $0.1 \mathrm{M} \mathrm{HClO}_{4}$ solution. (b) ECSA for various catalysts.
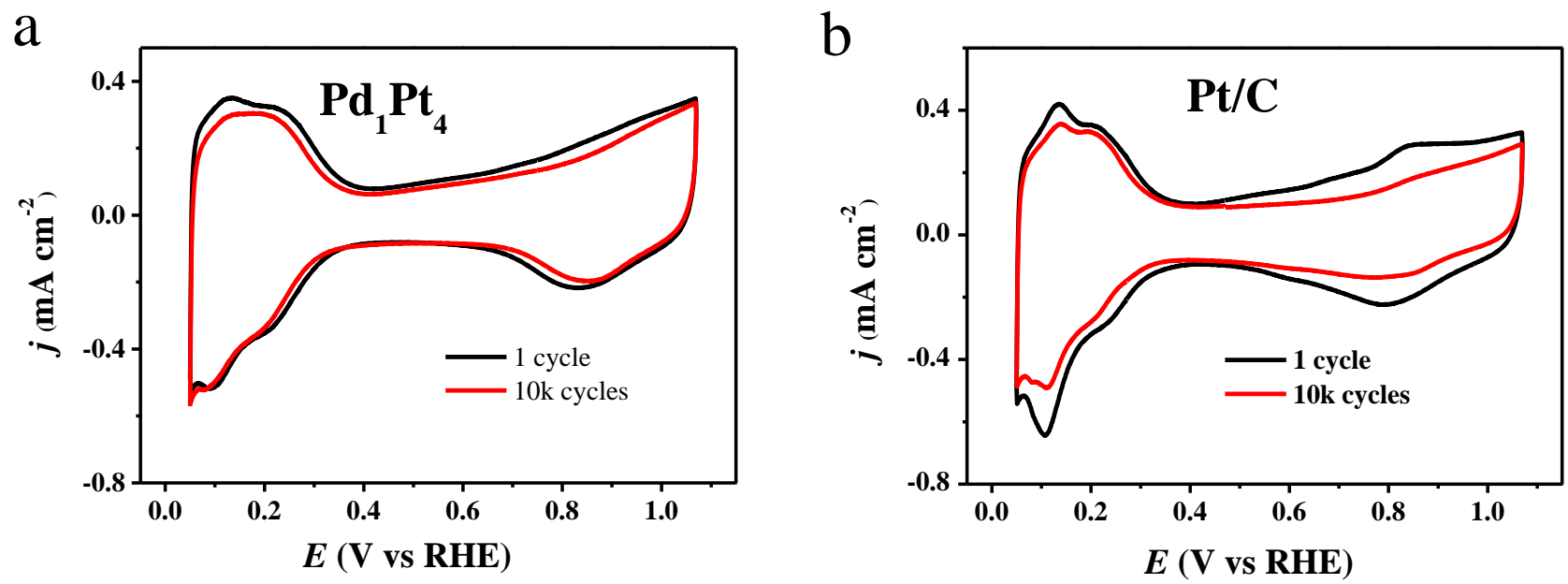

Figure S5. CVs of (a) $\mathrm{Pd}_{1} \mathrm{Pt}_{4}$ DNSs (b) $\mathrm{Pt} / \mathrm{C}$ before and after 10,000 potential cycles between 0.6 and $1.1 \mathrm{~V}$ versus $\mathrm{RHE}$ in $\mathrm{N}_{2}$-saturated $0.1 \mathrm{M} \mathrm{HClO}_{4}$ solution 

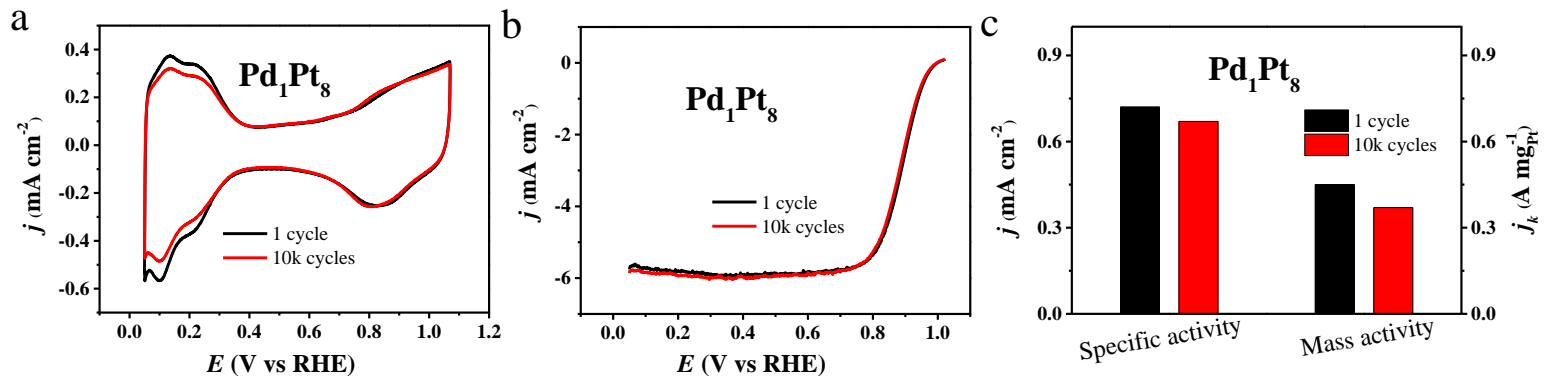

Figure S6 (a) CVs of $\mathrm{Pd}_{1} \mathrm{Pt}_{8} \mathrm{DNSs}$ before and after 10,000 potential cycles between 0.6 and 1.1 V versus $\mathrm{RHE}$ in $\mathrm{N}_{2}$-saturated $0.1 \mathrm{M} \mathrm{HClO}_{4}$ solution (b) ORR polarization curves of $\mathrm{Pd}_{1} \mathrm{Pt}_{16}$ DNSs catalyst before and after 10,000 potential cycles between 0.6 and $1.1 \mathrm{~V}$ versus RHE. (c) The histogram of specific activities and mass activities of $\mathrm{Pd}_{1} \mathrm{Pt}_{8} \mathrm{DNSs}_{\text {before and }}$ after 10,000 potential cycles between 0.6 and $1.1 \mathrm{~V}$ versus RHE.
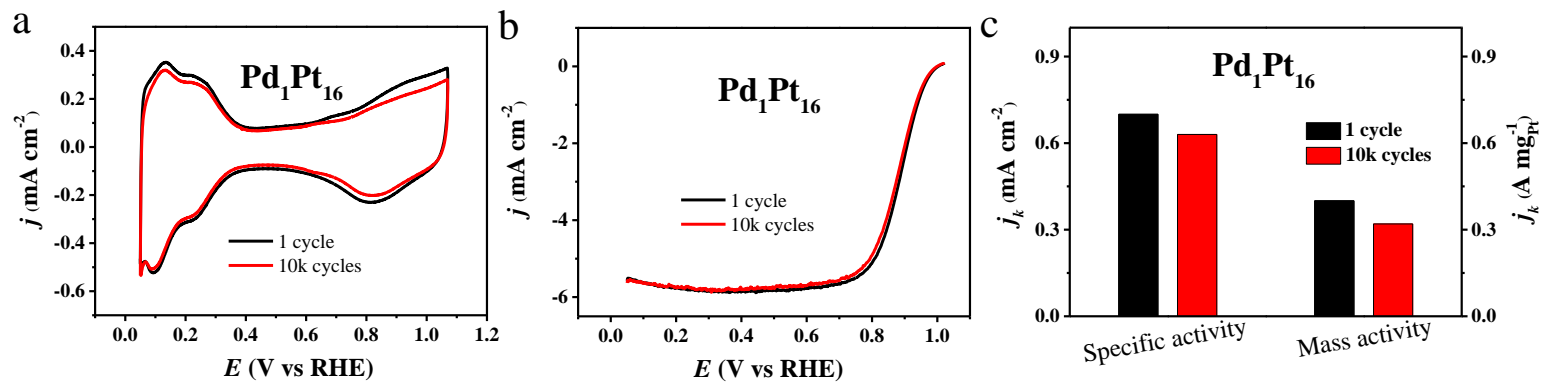

Figure $\mathrm{S} 7$ (a) CVs of $\mathrm{Pd}_{1} \mathrm{Pt}_{16}$ DNSs before and after 10,000 potential cycles between 0.6 and $1.1 \mathrm{~V}$ versus $\mathrm{RHE}$ in $\mathrm{N}_{2}$-saturated $0.1 \mathrm{M} \mathrm{HClO}_{4}$ solution (b) ORR polarization curves of $\mathrm{Pd}_{1} \mathrm{Pt}_{16} \mathrm{DNSs}$ catalyst before and after 10,000 potential cycles between 0.6 and $1.1 \mathrm{~V}$ versus RHE. (c) The histogram of specific activities and mass activities of $\mathrm{Pd}_{1} \mathrm{Pt}_{16} \mathrm{DNSs}$ before and after 10,000 potential cycles between 0.6 and $1.1 \mathrm{~V}$ versus RHE. 

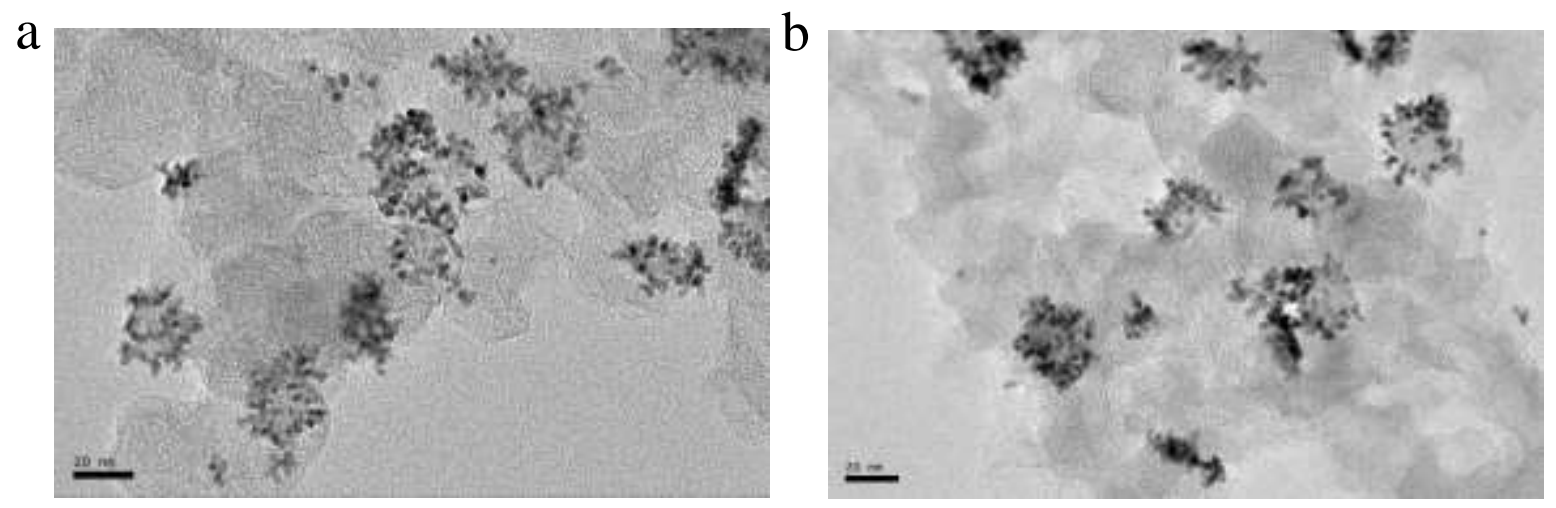

Figure S8 TEM images of $\mathrm{Pd}_{1} \mathrm{Pt}_{4}$ DNSs catalyst before (a), and after (b) $4000 \mathrm{~s}$ of durability test.
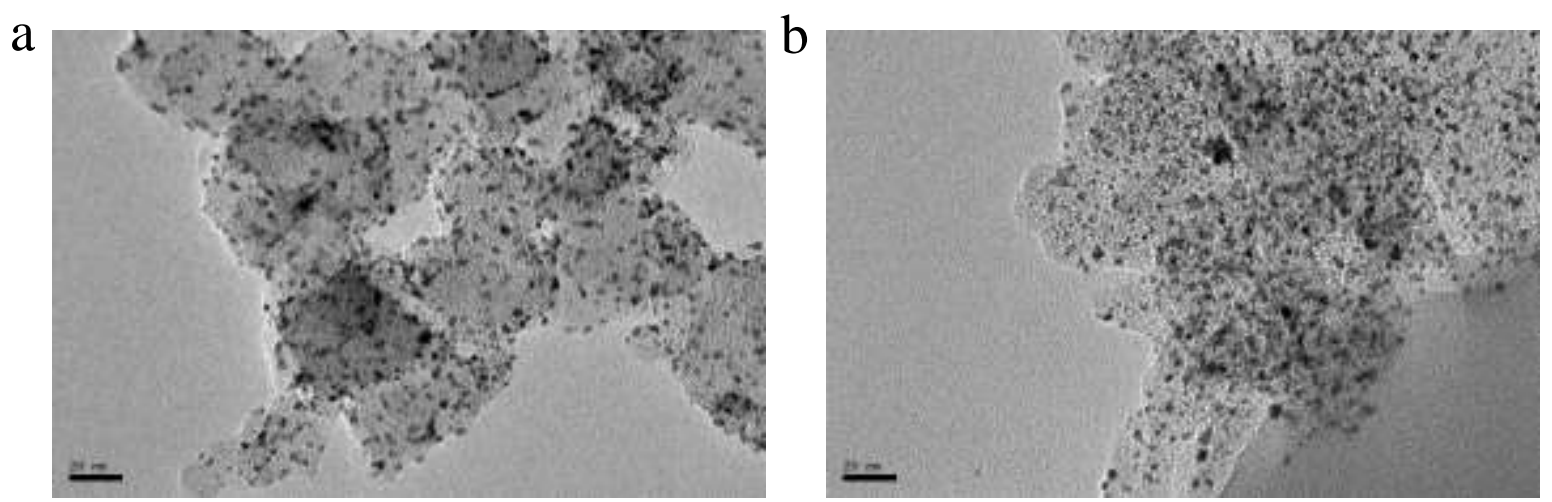

Figure S9 TEM images of Pt/C catalyst before (a), and after (b) $4000 \mathrm{~s}$ of durability test. 
Table S1 The synthesis of Pt-on-Pd DNSs with different compositions.

\begin{tabular}{ccccc}
\hline Pt-on-Pd DNSs & Pd NSs & $\mathrm{K}_{2} \mathrm{PtCl}_{4}(0.1 \mathrm{M})$ & $\mathrm{PVP}$ & Ascorbic acid \\
\hline $\mathrm{Pd}_{1} \mathrm{Pt}_{4}$ & $2.5 \mathrm{~mL}$ & $0.5 \mathrm{~mL}$ & $27.8 \mathrm{mg}$ & $52.8 \mathrm{mg}$ \\
$\mathrm{Pd}_{1} \mathrm{Pt}_{8}$ & $2.5 \mathrm{~mL}$ & $1.0 \mathrm{~mL}$ & $55.6 \mathrm{mg}$ & $105.6 \mathrm{mg}$ \\
$\mathrm{Pd}_{1} \mathrm{Pt}_{16}$ & $2.5 \mathrm{~mL}$ & $2.0 \mathrm{~mL}$ & $101.0 \mathrm{mg}$ & $211.2 \mathrm{mg}$ \\
\hline
\end{tabular}

Table S2 Comparison of electrocatalytic activities with different catalysts for ORR

\begin{tabular}{cccccc}
\hline Catalysts & $\begin{array}{c}\text { One-set potential } \\
(\mathrm{V} / \mathrm{RHE})\end{array}$ & $\begin{array}{c}\text { Half-wave potentials } \\
(\mathrm{V} / \mathrm{RHE})\end{array}$ & $\begin{array}{c}\mathrm{SA} \\
\left(\mathrm{mA} \mathrm{cm}{ }^{-2}\right)\end{array}$ & $\begin{array}{c}\mathrm{MA} \\
\left(\mathrm{A} \mathrm{mg}_{\mathrm{Pt}^{-1}}\right)\end{array}$ & $\begin{array}{c}\mathrm{MA} \\
\left(\mathrm{A} \mathrm{mg}_{\text {metal }}{ }^{-1}\right)\end{array}$ \\
\hline $\mathrm{Pd}_{1} \mathrm{Pt}_{4}$ & 0.998 & 0.887 & 0.74 & 0.53 & 0.47 \\
$\mathrm{Pd}_{1} \mathrm{Pt}_{8}$ & 1.000 & 0.887 & 0.72 & 0.45 & 0.42 \\
$\mathrm{Pd}_{1} \mathrm{Pt}_{16}$ & 1.003 & 0.883 & 0.72 & 0.4 & 0.39 \\
$\mathrm{Pt} / \mathrm{C}$ & 0.973 & 0.852 & 0.25 & 0.16 & 0.16 \\
\hline
\end{tabular}

Table S3 Comparison of the catalytic activities of various Pd-Pt bimetallic dendritic nanoparticles for ORR

\begin{tabular}{ccccc}
\hline Catalysts & $\begin{array}{c}\text { Specific activity } \\
\text { (at } 0.9 \mathrm{~V})\end{array}$ & $\begin{array}{c}\text { Mass activity } \\
\text { (at } 0.9 \mathrm{~V})\end{array}$ & ECSA & References \\
\hline $\begin{array}{c}\text { Pt-on-Pd Bimetallic } \\
\text { Heteronanostructures }\end{array}$ & $0.307 \mathrm{~mA} / \mathrm{cm}^{2}$ & --- & $37.3 \mathrm{~m}^{2} / \mathrm{g}$ & 1 \\
Pd-Pt nanodendrites & $0.424 \mathrm{~mA} / \mathrm{cm}^{2}$ & $0.241 \mathrm{~A} / \mathrm{mg}_{\mathrm{Pt}}$ & $57.1 \mathrm{~m}^{2} / \mathrm{g}$ & 2 \\
$\begin{array}{c}\text { Dendritic Bimetallic } \\
\text { Pt-Pd Nanoparticles }\end{array}$ & $0.258 \mathrm{~mA} / \mathrm{cm}^{2}$ & $0.139 \mathrm{~A} / \mathrm{mg}$ & $44.2 \mathrm{~m}^{2} / \mathrm{g}$ & 3 \\
$\begin{array}{c}\text { Pt-Pd nanodendrites } \\
\text { Pt-on-Pd dendritic } \\
\text { nanostructures }\end{array}$ & $0.435 \mathrm{~mA} / \mathrm{cm}^{2}$ & $0.402 \mathrm{~A} / \mathrm{mg}_{\mathrm{Pt}}$ & $92.4 \mathrm{~m}^{2} / \mathrm{g}$ & 4 \\
& $0.342 \mathrm{~mA} / \mathrm{cm}^{2}$ & $36.5 \mu \mathrm{A} / \mu \mathrm{g}$ & --- & 5 \\
Pt-on-Pd DNPs & $0.74 \mathrm{~mA} / \mathrm{cm}^{2}$ & $0.53 \mathrm{~A} / \mathrm{mg}_{\mathrm{Pt}}$ & $71.7 \mathrm{~m}^{2} / \mathrm{g}$ & This work \\
\hline
\end{tabular}


References

[1] Zhenmeng Peng, Hong Yang, Synthesis and Oxygen Reduction Electrocatalytic Property of Pt-on-Pd Bimetallic Heteronanostructures, J. Am. Chem. Soc., 2009, 131, 7542-7543

[2] Byungkwon Lim, Majiong Jiang, Pedro H. C. Camargo, Eun Chul Cho, Jing Tao, Xianmao Lu, Yimei Zhu, Younan Xia, Pd-Pt Bimetallic Nanodendrites with High Activity for Oxygen Reduction, Science, 2009, 324, 1302-1305

[3] Litai Sun, Hongjing Wang, Kamel Eid, Saad M. Alshehri, Victor Malgras, Yusuke Yamauchi, Liang Wang, One-Step Synthesis of Dendritic Bimetallic PtPd Nanoparticles on Reduced Graphene Oxide and Its Electrocatalytic Properties, Electrochim. Acta, 2016, 188, $845-851$

[4] Xiaoli Xiong, Wenhui Chen,Wang Wang, Jun Li, Shengli Chen, Pt-Pd Nanodendrites as Oxygen Reduction Catalyst in Polymer-Electrolyte-Membrane Fuel Cell, Int. J. Hydrogen. Energ., 2017, 42, 25234-25243

[5] Sourov Ghosh, Siniya Mondal, C. Retna Raj, Carbon Nanotube-Supported Dendritic Pt-on-Pd Nanostructures: Growth Mechanism and Electrocatalytic Activity towards Oxygen Reduction Reaction, J. Mater. Chem. A, 2014, 2, 2233-2239 